Revue internationale P.M.E.

Économie et gestion de la petite et moyenne entreprise

\title{
La crise du financement de la petite ou moyenne entreprise (PME) au Sénégal
}

\section{Coumba Ndoffène Diouf et Mohamed El Bachir Wade}

Volume 5, numéro 3-4, 1992

URI : https://id.erudit.org/iderudit/1008154ar

DOI : https://doi.org/10.7202/1008154ar

Aller au sommaire du numéro

Éditeur(s)

Presses de l'Université du Québec

ISSN

0776-5436 (imprimé)

1918-9699 (numérique)

Découvrir la revue

Citer cet article

Diouf, C. N. \& Wade, M. E. B. (1992). La crise du financement de la petite ou moyenne entreprise (PME) au Sénégal. Revue internationale P.M.E., 5(3-4), 49-60. https://doi.org/10.7202/1008154ar

\section{Résumé de l'article}

La question du financement de la petite ou moyenne entreprise (PME) a fait l'objet d'un débat passionné entre entrepreneurs et banquiers au Sénégal. Celui-ci découle d'une série de procédures de saisie-arrêt (portant sur des biens appartenant à des " opérateurs économiques » sénégalais) qui suscita beaucoup d'émotion dans l'opinion publique.

Cet article, basé sur un travail mené par des étudiants de troisième cycle entre 1986 et 1987 à Dakar, tente d'éclairer ce débat d'une part, à partir d'une double enquête auprès des protagonistes et, d'autre part, par l'analyse des bilans d'un échantillon de 32 entreprises sur une période de cinq ans correspondant au paroxysme de la crise.
Tous droits réservés @ Presses de l’Université du Québec, 1992
Ce document est protégé par la loi sur le droit d'auteur. L’utilisation des services d’Érudit (y compris la reproduction) est assujettie à sa politique d'utilisation que vous pouvez consulter en ligne.

https://apropos.erudit.org/fr/usagers/politique-dutilisation/ 


\title{
La crise du financement de la petite ou moyenne entreprise (PME) au Sénégal
}

\author{
Coumba Ndoffène DIOUF*

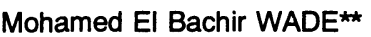 \\ Université Cheikh Anta Diop
}

\begin{abstract}
RÉSUMÉ
La question du financement de la petite ou moyenne entreprise (PME) a fait l'objet d'un débat passionné entre entrepreneurs et banquiers au Sénégal. Celui-ci découle d'une série de procédures de saisie-arrêt (portant sur des biens appartenant à des "opérateurs économiques" sénégalais) qui suscita beaucoup d'émotion dans l'opinion publique.

Cet article, basé sur un travail mené par des étudiants de troisième cycle entre 1986 et 1987 à Dakar, tente d'éclairer ce débat d'une part, à partir d'une double enquête auprès des protagonistes et, d'autre part, par l'analyse des bilans d'un échantillon de 32 entreprises sur une période de cinq ans correspondant au paroxysme de la crise.
\end{abstract}

\begin{abstract}
Small and medium-sized business finance provoked heated debate between Senegalese entrepreneurs and bankers after a series of much publicised garnishee orders had been issued against Senegalese operators.

This paper is based on research conducted by four doctoral students in 19861987. It aims at shedding new light on this debate on the basis of two surveys, one dealing with the protagonists of the dispute, the other with the financial statements of thirty two businesses over the five-year period of its duration.
\end{abstract}

* Coumba Ndoffène Diouf est assistant à l'Université CAD de Dakar et membre de l'équipe Partenariat du réseau entreprenariat de l'AUPELF-UREF. Il a travaillé à la création des PME au Sénégal et s'intéresse actuellement aux stratégies de survie et de développement qu'elles mettent en œuvre. Adresse : Faculté des sciences juridiques et économiques de l'Université Cheikh Anta Diop, Dakar.

** Mohamed El Bachir est également assistant à la Faculté des sciences juridiques et économiques de l'Université CAD de Dakar et membre de l'équipe Partenariat. Il s'intéresse particulièrement aux styles de gestion appliqués dans les entreprises et aux méthodes de contrôle. Adresse : Faculté des sciences juridiques et économiques de l'Université Cheikh Anta Diop, Dakar. 


\section{RESUMEN}

El tema de la financiación de la pequeña y mediana empresa (PME) ha sido objeto de un debate apasionado entre empresarios y banqueros en Senegal, resultado de una serie de trámites de embargo y de retención (de bienes pertenecientes a "operadores económicos" senegaleses) lo que ha sucitado el interés de la opinión pública.

Este artículo, basado en un trabajo llevado a cabo por estudiantes del tercer ciclo entre 1986 y 1987 en Dalcar, trata de aclarar este decate. Primero, a partir de una doble encuesta a los protagonistas, y segundo mediante el análisis de balances de una muestra de treinta y dos empresas durante un periodo de cinco años, correspondiente al paroxismo de la crisis.

\section{Introduction}

La question du financement de la petite ou moyenne entreprise (PME) au Sénégal a fait l'objet d'une polémique passionnée entre entrepreneurs et banquiers 1 .

L'éclosion et l'évolution de ce débat ont révélé une très profonde crise de financement de «l'entrepreneuriat national » en gestation. En effet, le point de départ de la polémique était avant tout la forte charge d'émotion créée par une série de procédures de saisie de biens appartenant aux premiers hommes d'affaires autochtones ${ }^{2}$ tentés par l'aventure industrielle au sein de petites et moyennes entreprises (PME).

Les deux principaux problèmes des PME industrielles soulevés sont d'ordre financier. Il y a d'une part l'absence de fonds de roulement (FR) et, d'autre part, l'insuffisance des fonds propres.

Les banquiers affirmaient que les entreprises vivaient « au jour le jour ", sans se soucier de cette marge de sécurité que constitue le fonds de roulement (FR); tandis que les entrepreneurs, eux, se plaignaient de leur refus, quasi systématique, de financer l'investissement en fonds de roulement sous prétexte de l'insuffisance des fonds propres des entreprises et des garanties qu'elles pouvaient offrir. Le cercle vicieux était ainsi bouclé. Et le. débat s'enlisait de plus en plus.

1. Ce débat s'est déroulé dans des séminaires, tables rondes et autres conférences ; les colonnes de la presse écrite même en font état très tôt. Citons par exemple : Le Soleil des 13 et 21 septembre 1982, et du 7 avril 1983 ; Takusaan du 17 avril 1983.

2. Ce sont ces hommes d'affaires qui ont, pour la plupart, profité de l'appui des pouvoirs politiques qu'on appelle les opérateurs économiques. 
En prenant un peu de recul, une équipe de quatre étudiants de troisième cycle a tenté de poser les termes exacts du problème pour trouver une base objective de résolution de cette crise. Il s'agissait surtout de faire le point sur le financement de la PME sénégalaise (industrielle notamment).

Avant de présenter plus nettement cette recherche, précisons d'abord le concept de PME. dans le contexte sénégalais.

Habituellement, pour définir une PME, on emploie des critères qualitatifs et des critères quantitatifs ${ }^{3}$. Au plan de l'opérationnalisation, les critères quantitatifs sont plus performants même si l'on peut montrer que les caractéristiques essentielles de la PME sont davantage qualitatives et structurelles que quantitatives.

Au Sénégal, trois définitions de la PME sont proposées à différents niveaux législatifs et réglementaires. Elles sont toutes marquées par le souci de la promotion des hommes d'affaires sénégalais. Mais, en dehors d'une condition qualitative de nationalité des propriétaires et dirigeants, elles reposent sur des critères quantitatifs.

Celle que nous avons utilisée pour la confection de l'échantillon de cette recherche est élaborée par la Société nationale d'encadrement et de promotion industrielle (SONEPI). Elle prend en considération :

- l'investissement (compris entre 5 et 250 millions de FCFA ${ }^{4}$ );

- les emplois permanents (entre 5 et 300 personnes);

- le chiffre d'affaires (compris entre 5 et 500 millions de FCFA).

3. Les critères qualitatifs reposent généralement :

- sur le lien entre l'entreprise et le propriétaire qui, en tant que principal gestionnaire, y pose son empreinte personnelle ;

- sur son rapport au marché ;

- sur son organisation centralisée qui dissocie très peu les sphères productive et domestique ;

- sur sa position vis-à-vis du marché financier (faible recours et accès limité au crédit bancaire);

- sur ses outils de gestion (on use peu de la comptabilité générale et surtout analytique et des autres méthodes de gestion).

Les critères quantitatifs, quant à eux, se réfèrent le plus souvent à l'effectif permanent employé, au chiffre d'affaires, à la valeur ajoutée, au montant de l'investissement réalisé, au niveau de l'encours bancaire, etc.

4. 1 franc $\mathrm{CFA}=0,02$ franc français. 
Ce choix s'explique par une plus grande opérationnalisation de cette définition. En effet, ces critères et leurs seuils sont déclinés en fonction des différents secteurs, selon qu'ils sont capitalistiques ou utilisateurs de main-d'œuvre.

La méthodologie adoptée pour approcher le problème du financement de la PME sénégalaise repose sur une double enquête par questionnaire et l'exploitation de documents comptables d'un échantillon de 32 entreprises.

La première enquête a porté sur un échantillon qui, par sa composition, est assez représentatif de l'ensemble des PME industrielles (PMEI) implantées dans la région de la capitale du Sénégal. Elle s'est intéressée au financement des PMEI à divers moments de leur vie (création, extension, activités courantes) et sur divers horizons (long, moyen et court terme).

La seconde enquête s'est adressée à la totalité des banques de la place. Son but était de faire l'état des structures bancaires existantes et des relations qu'elles entretiennent avec les PME.

Ces deux enquêtes ont obtenu des taux de réponses jugés satisfaisants. En effet, on a recueilli auprès des entreprises 27 questionnaires totalement exploitables et 5 autres partiellement remplis. Sur les 15 banques qui existaient au moment de l'enquête, 7 ont répondu à nos questions.

Les résultats du dépouillement peuvent être analysés en distinguant deux niveaux dans la crise du financement des PME : le financement à long et à moyen terme (LMT) (1) et le financement de l'exploitation (2).

\section{La crise du financement à LMT}

L'étude du financement à LMT de la PMEI sénégalaise fait apparaître plus qu'un malaise dans la relation qu'elle entretient avec les banques, une crise structurelle. Celle-ci tient aussi bien de l'insuffisance du financement propre que de la quasi-absence de crédit bancaire à long et moyen terme. Elle peut être analysée en distinguant le financement de la création du financement de la PME en activité.

\subsection{Le financement de la création de PME}

La création d'entreprise est un processus suivant lequel une personne qui a mûri une idée, la traduit en projet qu'elle s'évertue ensuite à réaliser. Pour ce faire, il lui faut apporter des fonds propres et rechercher (s'il y a lieu) des crédits complémentaires en vue de mettre en place les équipements et matières nécessaires à l'exploitation. 
Le financement de la création est un problème important et délicat. La réponse qui lui est donnée détermine, dans une large mesure, le devenir de l'entreprise et sa capacité à maîtriser son exploitation.

Au Sénégal, cette question est loin d'avoir une solution satisfaisante ${ }^{5}$. Il y a une pénurie de capitaux qui fait que, par exemple, à la SONEPI, on disposait de 400 projets entièrement étudiés et toujours en attente de réalisation, faute de financement. Cette pénurie de capitaux touche aussi bien les entrepreneurs individuels que les intermédiaires financiers. Elle est consécutive à la faiblesse de l'accumulation et à celle du drainage de l'épargne dans les structures formalisées. Elle a sûrement influencé, et fortement, le comportement des banquiers face à leurs clients ordinaires ${ }^{6}$, lequel est dominé par la prudence excessive. C'est ainsi qu'on peut noter (dans le tableau 1 ci-dessous) que la principale cause de refus ou d'annulation de crédit évoquée par les banques est l'insuffisance des fonds propres ( $46 \%$ des réponses). Leur niveau ainsi que les autres garanties matérielles importent plus que tout ; on s'intéresse très peu à la valeur intrinsèque du projet à financer ou aux qualités de son promoteur.

TABLEAU 1

Les causes de refus de crédit

\begin{tabular}{lcccccccc}
\hline Cause & $\begin{array}{c}\text { Défaut } \\
\text { d'auto- } \\
\text { risation }\end{array}$ & $\begin{array}{c}\text { Défaut } \\
\text { de } \\
\text { partenaire } \\
\text { technique }\end{array}$ & $\begin{array}{c}\text { Défaut } \\
\text { de } \\
\text { financier }\end{array}$ & $\begin{array}{c}\text { Insuffí- } \\
\text { sance } \\
\text { des FP }\end{array}$ & $\begin{array}{c}\text { Insuffi- } \\
\text { sance } \\
\text { de } \\
\text { garantie }\end{array}$ & $\begin{array}{c}\text { Autres } \\
\text { problèmes } \\
\text { adminis- } \\
\text { tratif }\end{array}$ & $\begin{array}{c}\text { Glissement } \\
\text { du } \\
\text { marché }\end{array}$ & $\begin{array}{c}\text { Abandon } \\
\text { créateur }\end{array}$ \\
\hline Fréquence & 0 & $8 \%$ & $15 \%$ & $46 \%$ & $8 \%$ & $15 \%$ & $8 \%$ & 0 \\
\hline
\end{tabular}

De toute façon, les banquiers ne semblent pas assez outillés pour apprécier et faire face à certains types de besoins particuliers. Aucune des banques privées de la place n'a, en effet, de programmes spéciaux pour la création d'entreprise ou pour le financement de la PME. Ces banques ne font aucune distinction entre de tels besoins de financement et les autres. Quant aux banques de développement, elles ont un intérêt pour la création et les PME qui se limite surtout à la gestion des lignes de crédit accordées par l'État pour sa politique de promotion des PME qui y sont domiciliées.

5. Le crédit accordé au gouvernement par la $B A D$ pour financer un Fonds de promotion économique contribuera, partiellement sans doute, à la résolution de ce problème.

6. Nous entendons par là les clients qui n'ont pas de protecteurs politiques ou religieux. En effet, les bénéficiaires de passe-droits sont prioritaires; et généralement, on ne leur demande pas de remplir certaines conditions. 
TABleau 2

Existence de division ou organismes spécialisés selon le type de banque

\begin{tabular}{lccccc}
\hline $\begin{array}{l}\text { Programme } \\
\text { ou Division }\end{array}$ & $\begin{array}{c}\text { Division } \\
\text { industrielle }\end{array}$ & $\begin{array}{c}\text { Programme } \\
\text { PME }\end{array}$ & $\begin{array}{c}\text { Programme } \\
\text { création }\end{array}$ & $\begin{array}{c}\text { Programme } \\
\text { fonds de } \\
\text { roulement }\end{array}$ & $\begin{array}{c}\text { Division } \\
\text { études }\end{array}$ \\
\hline Banques de & 2 & 3 & 2 & 1 & 2 \\
développement & 2 & 0 & 0 & 0 & 0 \\
Banques privées & 0 & 0 & & & \\
\hline
\end{tabular}

La pénurie de ressources a entrainé la sous-capitalisation des entreprises qui se manifeste de deux manières:

- les entreprises sont sous-équipées et fonctionnent au détriment de la qualité avec le minimum de matériel nécessaire ;

- le fonds de roulement est quasi inexistant pour bon nombre d'activités, les banques refusant de financer l'investissement en fonds de roulement.

Au total, on peut retenir que les difficultés de financement de la création procèdent d'une crise structurelle du financement à LMT dans une « économie de consommation » où la faiblesse de l'épargne collectée restreint grandement l'intermédiation bancaire.

\subsection{Le financement à LMT de la PME en activité}

Le financement à LMT a pour vocation de permettre l'acquisition des immobilisations et la mise en place d'un fonds de roulement suffisant. Il mobilise des capitaux provenant essentiellement de deux sources : les fonds propres de l'entreprise et l'endettement auprès des banques.

Au Sénégal, on peut constater que les PME se financent surtout par fonds propres : près de $73 \%$ des entreprises de notré échantillon n'ont pas de dette à LMT.

L'analyse du ratio de capacité d'endettement durant la période allant de 1981 à 1985 montre que la plupart de nos entreprises n'ont pas utilisé pleinement leur capacité d'endettement (dettes totales/capitaux propres). S'agitil là d'un choix délibéré de politique de financement ?

L'évolution que décrit le tableau 3 nous incline à répondre non. 
TABleau 3

PME dont la capacité d'endettement est inférieure à $50 \%$

\begin{tabular}{llllll}
\hline Année & 1981 & 1982 & 1983 & 1984 & 1985 \\
\hline Fréquence & $18 \%$ & $23 \%$ & $33 \%$ & $36 \%$ & $47 \%$ \\
\hline
\end{tabular}

Il ressort de ce tableau qu'il y a une tendance croissante à l'endettement des entreprises, ce qui traduit indiscutablement un accroissement de leurs besoins de financement (à long et moyen terme notamment). En effet, dans la même période, on peut constater un problème aigu de fonds de roulement qui concernent plus d'un tiers des PME constituant notre échantillon (tableau 4).

TABLEAU 4

Fréquence des entreprises ayant un FR négatif

\begin{tabular}{lccccc}
\hline Année & 1981 & 1982 & 1983 & 1984 & 1985 \\
\hline Fréquence & $40 \%$ & $30 \%$ & $33,33 \%$ & $36,36 \%$ & $33,33 \%$ \\
\hline
\end{tabular}

L'analyse du financement de l'exploitation ci-après nous permettra d'apprécier les palliatifs de la négativité du fonds de roulement qui sont employés. Mais on peut noter d'ores et déjà qu'ils pèsent fortement sur la rentabilité et augmentent les risques d'exploitation, puisque les garanties données sont réalisées sans délai en cas de défaillance.

En effet, depuis le début des années 80 , la politique monétaire appliquée a entraîné un renchérissement du crédit du fait de son encadrement. Poursuivant un objectif de contrôle de la masse monétaire, la Banque Centrale a abaissé le plafond de ses concours au Trésor public et aux banques primaires tout en relevant son taux d'escompte de base. Par conséquent, les rares ressources disponibles sont drainées vers le financement du court terme, comme le montre le tableau 5 ci-dessous pour la période allant du $1^{\text {er }}$ juillet 1983 au $1^{\text {er }}$ juillet 1984 , par exemple.

TABLEAU 5

Répartition du crédit à l'économie du 1/7/83 au 1/7/84

\begin{tabular}{lccc}
\hline Type de crédit & Court terme & Moyen terme & Long terme \\
\hline Pourcentage & $89,99 \%$ & $9,45 \%$ & $0,56 \%$ \\
\hline
\end{tabular}


Ce tableau illustre bien la boutade d'un banquier que nous avons rencontré : «Au Sénégal, le moyen terme existe peut-être, mais le long terme n'existe pas !»

Il exprime également, autant que les conditions de crédit, le très grand souci de sécurité qui anime les banquiers.

Pour tout crédit à LMT, il semble qu'on demande systématiquement une hypothèque avec en plus, soit un aval, soit un cautionnement : la valeur de garantie demandée ${ }^{7}$ dépasse toujours largement le montant du crédit (de 50 à $100 \%$ selon les sources).

L'examen de la répartition sectorielle du crédit à l'économie confirme bien ce souci de sécurité, car il montre que celui-ci est dirigé en priorité vers le secteur commercial, le secteur industriel n'ayant recueilli durant cette période que $31,94 \%$ du crédit à CT et 3,17\% du crédit à long et moyen terme.

Les tableaux 3 et 5 (évolution de la capacité d'endettement dans l'échantillon et du crédit à l'économie) montrent que la conjoncture économique et les conditions rigoureuses que les banquiers exigent pour l'octroi de crédit n'ont pas dissuadé les entreprises d'emprunter davantage, ce qui nous laisse supposer l'existence d'importants besoins de financement à satisfaire.

Au total, nous avons montré que les PMEI au Sénégal souffrent d'une pénurie de capitaux à LMT à tous les stades de leur développement. À la création, par exemple, elles ont beaucoup de difficulté à financer leurs équipements et surtout leur fonds de roulement. Elles ne peuvent que rarement et marginalement compter sur le système bancaire pour leur expansion. Les banques sénégalaises favorisent les activités commerciales au détriment des activités industrielles ; elles n'acceptent pratiquement que des crédits à court terme. Un tel comportement paraît avoir un impact négatif sur les PMEI qui sont obligées d'utiliser un financement qu'elles savent inapproprié pour couvrir certains de leurs besoins. L'examen du financement de leur exploitation permet de mettre en évidence quelques-unes de ces distorsions.

\section{Les distorsions dans le financement de l'exploitation}

La tension qui pèse sur le financement de l'activité de nos PMEI est déjà annoncée par la fréquence des fonds de roulement négatifs. Pour la mettre en

7. D'après un banquier, « $120 \%$ est un taux minimum qui permet de préserver une certaine marge de réalisation ». 
relief, nous pouvons analyser les moyens utilisés pour couvrir les besoins de financement de l'exploitation (endettement à CT) et la trésorerie.

\subsection{L'endettement à court terme}

La longueur du cycle d'exploitation, la vitesse de rotation des stocks ainsi que la politique de crédit-clients engendrent un besoin de financement à court terme qui est généralement couvert par des concours bancaires à CT et des créditsfournisseurs.

Pour ce qui est de notre échantillon, les dettes bancaires à CT représentent 10 à $30 \%$ de l'endettement à CT total contre 35 à $48 \%$ pour les dettes à l'égard des fournisseurs. Les dettes envers l'État ou les organismes sociaux (sécurité sociale, caisse de retraite), ainsi que les rémunérations dues au personnel, constituent le reste du passif à court terme. Cette répartition moyenne des dettes à CT confirme les difficultés particulières d'accès au crédit bancaire à CT que rencontrent les petites et moyennes entreprises.

D'ailleurs, $64 \%$ des entrepreneurs que nous avons rencontrés soulignent les efforts qu'il leur faut déployer pour obtenir un crédit bancaire, même à court terme (qui est jugé inadapté à leurs besoins par la moitié d'entre eux).

Différentes analyses statistiques (études de corrélation) montrent que les dettes bancaires sont utilisées par les entreprises (qui y ont accès) pour préfinancer des contrats en l'absence de fonds de roulement.

D'autre part, le crédit commercial interentreprises ne profite qu'à $29 \%$ de l'échantillon. En effet, la majorité de nos entreprises laissent à leurs clients un délai supérieur à celui qu'elles obtiennent de leurs fournisseurs. Seulement $17,65 \%$ des entreprises ont des créances-clients inférieures à 80 jours. Pour $52,9 \%$ des entreprises, la durée des créances-clients est comprise entre 131 et 452 jours alors que leur cycle de production couvre à peine trois mois. En fait, il faut souligner que les entreprises subissent plutôt la longueur des délaisclients qu'elles ne les consentent au moment de la vente. En effet, face aux difficultés de financement qui les touchent toutes, elles avaient souscrit à un "gentleman-agreement », à une sorte de règle implicite de non-sanction de l'insolvabilité. Ainsi, comme le montrent les différents tests de corrélation entre les créances-clients et les crédits-fournisseurs, chacun essaie de supporter les délais de recouvrement imposés par sa clientèle en transférant le fardeau à ses fournisseurs ou, le cas échéant, en ayant recours à la trésorerie. 


\title{
2.2. Le recours massif à la trésorerie passive
}

\author{
TABleaU 6 \\ Répartition des PME selon leur trésorerie \\ (en millions)
}

\begin{tabular}{lccccc}
\hline $\begin{array}{l}\text { Niveau de } \\
\text { trésorerie/Année }\end{array}$ & $\mathbf{1 9 8 1}$ & $\mathbf{1 9 8 2}$ & $\mathbf{1 9 8 3}$ & $\mathbf{1 9 8 4}$ & $\mathbf{1 9 8 5}$ \\
\hline Plus de 100 & 1 & 0 & 0 & 0 & 0 \\
Entre 100 et 50 & 1 & 1 & 0 & 2 & 0 \\
Entre 50 et 25 & 3 & 4 & 4 & 0 & 2 \\
Entre 25 et 5 & 3 & 4 & 5 & 9 & 7 \\
Entre 5 et 0 & 3 & 2 & 5 & 2 & 1 \\
Entre 0 et -5 & 1 & 5 & 2 & 3 & 6 \\
Entre -5 et -25 & 6 & 4 & 10 & 7 & 7 \\
Entre -25 et -50 & 2 & 3 & 2 & 5 & 4 \\
Entre -50 et -100 & 4 & 4 & 4 & 3 & 2 \\
Au delà de -100 & 0 & 2 & 0 & 1 & 0 \\
\hline
\end{tabular}

L'examen de la liquidité de nos entreprises fait apparaître une tendance à la hausse des cas de trésorerie négative : $54 \%$ en $1981,62 \%$ en $1982,56 \%$ en $1983,62 \%$ en $1984,65 \%$ en 1985.

Une analyse plus fine permet d'isoler cinq groupes de PME.

Le premier groupe, le plus important, représentant $44 \%$ de l'effectif, comprend les entreprises ayant une trésorerie structurellement déficitaire.

Le second groupe, soit $22 \%$ de l'échantillon, réunit celles dont la trésorerie s'est dégradée au cours de la période, passant d'une position positive à un déficit.

Les entreprises avec une trésorerie structurellement excédentaire forment le troisième groupe en importance (19\%).

Le quatrième groupe est celui des entreprises qui ont progressivement résorbé leur déficit de trésorerie ; il représente $9 \%$ de l'échantillon.

Enfin, il y a les entreprises dont la trésorerie a eu un comportement erratique, alternant déficits et excédents ; elles forment les $6 \%$ restants.

Au total, l'examen de la trésorerie de notre échantillon apporte une confirmation tout à fait claire de nos différentes constatations antérieures, à partir 
de la relation fondamentale qui lie la trésorerie, le fonds de roulement et le besoin en fonds de roulement. En effet, un fonds de roulement inexistant ou, en tout cas, insuffisant pour couvrir les besoins en fonds de roulement, entraine une trésorerie négative.

C'est cette situation qui était, en fait, à l'origine de la querelle entre les hommes d'affaires et les banquiers, les entrepreneurs appréciant mal le fait de devoir consacrer beaucoup de temps et d'efforts à la négociation de recours à la trésorerie, en définitive peu adaptés à leurs besoins, et les banquiers étant agacés par la métamorphose de facto de leur intervention qui d'exceptionnelle devient permanente tant la reconduction est à chaque fois sollicitée.

De part et d'autre, on avait donc conscience de la vulnérabilité qui découlait de cet état de fait, et l'on pouvait donc mesurer les dangers qu'engendrait la Nouvelle Politique Macroéconomique. Effectivement, avec la mise en œuvre d'un programme d'ajustement structurel à partir de 1985, la polémique connut son épilogue.

\section{Conclusion}

Dès 1986, les effets de la politique d'assainissement monétaire furent ressentis. Certaines banques, notamment celles du secteur parapublic, commençaient à connaître des problèmes de trésorerie, et ne pouvaient plus ni payer à vue tous les chèques provisionnés à leurs guichets, ni faire face à la compensation.

La restructuration bancaire qui s'ensuivit a eu comme principaux effets :

- la disparition des services bancaires destinés à l'industrie et aux PME, du fait de la liquidation des banques de développement qui en étaient offreurs ;

- la remise en cause de la règle implicite de non-sanction de la nonliquidité ;

- le gel, pendant plus de trois ans, des avoirs de certaines entreprises dans ces banques.

Il en résulta un vide, donc un surcroît de difficultés pour les PME, lequel ne connaîtra un début de solution qu'avec l'avènement du Fonds de promotion économique (FPE) en décembre 1991.

En fait, le différend entre les banquiers et les hommes d'affaires apparaît comme une querelle autour de la règle de non-sanction de l'insolvabilité (pour ou contre sa remise en cause partielle au détriment de ceux qui ont le plus faible pouvoir de négociation). 
Le transfert du patrimoine des banques liquidées à la Société nationale de recouvrement montra en effet qu'elles avaient surtout souffert de l'influence des pouvoirs politiques et religieux dans leur gestion ${ }^{8}$, ce qui a conduit à leur faillite.

\section{Bibliographie}

Banque et Monnale (1975), $n^{\circ}$ 236, février.

CiBerT, A. (1985), « Les P.M.I. en France aujourd'hui », APRODI

CIBERT, A., « Perspectives », Revue internationale P.M.E., vol. $2, \mathrm{n}^{\circ} 2$.

CisSE, I. (1984), Approche statistique de la P.M.E. au Sénégal, SES-SES/PME, SONEPI, Dakar.

Drouf, C.N. (1987), « Le financement de la création d'entreprises au Sénégal. Créateurs et Banquiers peuvent-ils s'entendre? Le cas du Sénégal », Mémoire de D.E.A. ès sciences de gestion, Dakar.

HIRIGOYen, G. (1981), « Caractéristiques des M.E.I. en France », Banque, n 408, juillet/ août.

SALL, M.B. (1987), « La gestion du crédit-clients dans les P.M.I. sénégalaises », Mémoire de D.E.A. ès sciences de gestion, Dakar.

SECK, A.A.H. (1988), « Le problème de l'insuffisance des fonds propres dans les P.M.I. Sénégalaises », Document inédit, Dakar.

SENE, P.M. (1988), «La problématique du financement de la P.M.I. au Sénégal », Mémoire de D.E.A. ès sciences de gestion, Dakar.

8. Ces banques avaient accordé des crédits de complaisance à des politiciens, des marabouts et autres chefs coutumiers qui ont été incapables de les rembourser; d'où le problème des « gros débiteurs » qui est aujourd'hui encore d'actualité. 